\title{
Alfabetización académica chilena: revisión de investigaciones de una década*
}

\author{
Academic Literacy in Chile: A Decade of Research Reviews
}

\author{
Lina Calle-Arango ${ }^{* *}$, Natalia Ávila-Reyes ${ }^{* * *}$
}

\section{RESUMEN}

Esta revisión bibliográfica profundiza en el estado de desarrollo y tendencias de los estudios sobre alfabetización académica en Chile. Se analizan las investigaciones empíricas chilenas publicadas durante una década en revistas indexadas en WoS y Scopus. Los 24 estudios encontrados sugieren una consolidación del campo, caracterizado por investigaciones en ciclos de pregrado, de estudiantes de primero y último año, muestras relativamente pequeñas, diseños cualitativos, y uso de entrevistas y grupos focales como técnicas frecuentes de recolección de datos. Asimismo, se corroboró lo señalado en estudios previos respecto a dos grandes tendencias investigativas: una cognitiva, y otra sociocognitiva. A partir de estos datos, se ofrecen consideraciones finales que caracterizan el campo y proyectan su entrada en las conversaciones internacionales de la disciplina.

\section{ABSTRACT}

This bibliographic review delves into the state of development and trends in academic literacy studies in Chile. It analyzes the Chilean empirical research published over the span of one decade in journals indexed in WoS and Scopus. The 24 studies identified suggest a consolidation of the field, characterized by research in undergraduate cycles, first and last year students, relatively small samples, qualitative designs, and the use of inter-
Palabras clave:

Alfabetización académica, escritura, estudios empíricos, escritura académica.

Keywords:

Academic literacy, writing, empirical research, academic writing

\footnotetext{
* $\quad$ Este artículo forma parte del proyecto FONDECYT INICIACIÓN 11170723. “Transformaciones letradas: un estudio longitudinal en escritores diversos". Pontificia Universidad Católica de Chile

** Colombiana. Máster en Edición de la Universidad de Salamanca, España. Doctoranda en Educación Pontificia Universidad Católica de Chile. 1calle@uc.cl

*** Chilena. Doctora en Educación de la University of California Santa Barbara, Estados Unidos. Académica de la Pontificia Universidad Católica de Chile. naavila@uc.cl
} 
Alfabetización académica chilena: revisión de investigaciones | Arango y Ávila

views and focus groups as common data collection techniques. In addition, previous studies corroborated two major research trends, namely, a cognitive trend and a sociocognitive one. Based on this data, final considerations are offered to characterize the field and project its penetration into international conversations on the discipline. 


\section{Introducción}

Los estudios de la escritura en Educación Superior en América Latina, referidos comúnmente como alfabetización académica, comenzaron a desarrollarse con gran fuerza en la región al igual que en otros lugares del mundo durante las últimas dos décadas (Bazerman, Dean, Early, Lunsford, Null, \& Stansell, 2012). En el caso de Latinoamérica, el interés de las instituciones de educación superior en las prácticas de lectura y escritura se ha incrementado notoriamente (Bazerman, Ávila, Bork, Poliseli-Corrêa, Cristovão, Tapia-Ladino \& Narváez-Cardona, 2016) debido a factores como la masificación del ingreso a la formación superior hacia fines del siglo XX (Pérez y Natale, 2017; Navarro, 2017), el giro hacia las pedagogías universitarias centradas en los estudiantes (González, 2015), o las políticas de aseguramiento de calidad y los regímenes de acreditación en la región (Ávila, González y Peñaloza, 2013). Este espacio disciplinar de práctica e investigación fue definido programáticamente por Paula Carlino (2003) como "alfabetización académica", o "el conjunto de nociones y estrategias necesarias para participar en la cultura discursiva de las disciplinas así como en las actividades de producción y análisis de textos requeridas para aprender en la universidad" (p.410).

Si bien la preocupación por formación en escritura en la universidad tiene antecedentes en los primeros movimientos de expansión universitaria en Argentina (Pereira, 2006), recién durante la década del 2000 se instalan en la región una serie de iniciativas que abarcan propuestas educativas, cursos propedéuticos, centros de escritura y elaboración de materiales y manuales de escritura (Ávila, González y Peñaloza, 2013; Natale, 2013; Molina, 2014; Navarro, 2014).

Desde inicios de esta década, la aparición de volúmenes monográficos y el aumento de estudios de metaanálisis disciplinar atestiguan un interés por sistematizar este trabajo. Por ejemplo, la revisión de Ortiz (2011) identificó dos enfoques dentro de estos estudios: uno cognitivo y otro sociocognitivo. Un análisis sobre la producción académica de la disciplina publicada entre 2001 y 2015 en cuatro revistas de Latinoamérica confirma una variedad y mistura de aproximaciones y escuelas teóricas, así como dos grandes tendencias a la hora de definir el objeto de estudio 1) estudiar la lectura y escritura estudiantiles y su enseñanza en un paradigma ecléctico, pero interesado en las pedagogías; o 2) 
estudiar el discurso académico como producto, en un paradigma más cercano al análisis lingüístico (Navarro, Ávila, Tapia Ladino, Cristivao, Moritz, Narváez y Bazerman, 2016). En este estudio, también se encontró una interesante predominancia de artículos empíricos por sobre teóricos o experienciales $(70,4 \%)$, concentrados fundamentalmente en las revistas estudiadas de Argentina y Chile. Si bien la denominación "alfabetización académica" continúa siendo el principal nombre dado al campo, otros textos han comenzado a referirse de manera más amplia a los "estudios de la escritura en educación superior", en un intento por rescatar los énfasis tanto discursivos como pedagógicos (Navarro et al., 2016; Tapia-Ladino, Ávila Reyes, Navarro, \& Bazerman, 2016).

Otros estudios (Bazerman et al, 2016; Tapia-Ladino et al., 2016; Ávila, 2017) han buscado relevar las perspectivas teóricas y epistemológicas subyacentes a los estudios latinoamericanos sobre las prácticas de lectura y escritura en la educación superior. Sus resultados confirman la consolidación de este interés a lo largo de la región, aunque también relevan la ambigüedad, e incluso la disputa de identidad epistemológica y disciplinaria de sus dos tradiciones de origen: la lingüística y los estudios educativos.

Los trabajos que rastrean las identidades intelectuales, epistemologías, paradigmas y prácticas de investigación, han sido frecuentes en los estudios sobre la escritura en otros lugares del mundo, ya sea en el ámbito norteamericano (Nystrand, Greeene, Wielmelt, 1993; Prior \& Thorne, 2014) en Inglaterra (Lillis y Scott, 2007) o en la tradición española (Castelló, 2002). Este tipo de trabajos contribuye a conceptualizar disciplinarmente el campo de estudio, y a sistematizar el conocimiento desarrollado a lo largo de las décadas de práctica, lo que permite comprender más claramente la naturaleza de la comunidad disciplinar emergente. En el caso de Latinoamérica podemos dar cuenta de numerosos esfuerzos como los recién reseñados (Navarro et al., 2016; Tapia Ladino et al., 2016; Bazerman et al., 2016; Ávila, 2017), pero se hace necesario aún estudiar las configuraciones disciplinares de manera más específica en los diferentes países que producen conocimiento en el área. Contar con una sistematización del conocimiento acumulado es beneficioso para identificar vacíos de conocimiento en el área, conocer los hallazgos de las tradiciones con las que se dialoga y, en suma, consolidar una investigación regional de calidad. 
Junto con Argentina, Chile sobresale en la región por poseer un robusto cuerpo de investigadores e instituciones interesados en participar de estas discusiones y contribuir a un diálogo local, pero también internacional (Ávila Reyes, 2018). A modo de ejemplo, al buscar las palabras clave escritura en educación superior en la base de datos Web of Science, Chile es el primer país latinoamericano hispanoparlante en aparecer en la lista, en el número 22. Desde la misma base de datos, la Pontificia Universidad Católica de Chile, la Pontificia Universidad Católica de Valparaíso y la Universidad de Chile aportan predominantemente las filiaciones. Es necesario, no obstante, reconocer una sobrerrepresentación del país en este índice debido a la aspiración preferente por índices bibliográficos mainstream, debido a las presiones por generar indicadores de competitividad universitaria. Este requisito, que plantea fuertes desafíos para una participación igualitaria de los académicos hispanohablentes en un espacio hegemonizado por publicaciones en inglés (Curry \& Lillis, 2017), permite al mismo tiempo contar con un indicador externo para localizar y dimensionar el aumento en la publicación chilena en este tema.

El presente estudio propone una revisión sistemática de la literatura para analizar las investigaciones empíricas chilenas publicadas durante la última década en revistas indexadas, para ahondar en el estado de desarrollo en Chile y caracterizar las investigaciones nacionales. La inclinación por trabajos empíricos se debe a la importancia que desempeñan en la agregabilidad del conocimiento al interior de una disciplina (Haswell, 2005). La decisión de estudiar las bases de datos de Web of Science y Scopus radica en el alto valor que tiene este tipo de publicaciones en el sistema científico nacional, tanto en las normas de calificación y promoción académica de las universidades como en la evaluación de productividad en fuentes de financiamiento externo. En efecto, en los grupos de estudio de Lingüística y de Educación del Fondo Nacional de Desarrollo Científico y Tecnológico de Chile, WoS y Scopus constituyen las indexaciones más altamente valoradas (Comisión Nacional de Ciencia y Tecnología, s/f). En síntesis, en las siguientes páginas se analizan descriptivamente las prácticas investigativas y se realiza un examen crítico de los textos explorados, reconociendo su aporte científico y su enorme valor en la consolidación del campo disciplinar en el país, con el objetivo de caracterizar las 
configuraciones epistemológicas que emergen y detectar brechas de conocimiento.

\section{Metodología}

Se realizó una búsqueda sistemática en las bases de datos de Web of Science y Scopus en un ciclo de diez años (2008-2018) mediante la combinación de la palabra clave higher education con (i) academic writing, (ii) academic genre, (iii) academic literacy y (iv) writing. Esta búsqueda fue actualizada en abril de 2019. La selección respondió a dos criterios: (i) investigaciones empíricas; y (ii) estudios realizados en instituciones de educación superior chilenas que estuvieran focalizados en la escritura académica de este ciclo formativo en primera lengua. Se descartaron artículos teóricos, propuestas metodológicas de enseñanza, metaanálisis e investigaciones en las que la escritura o los escritores no fueran objeto central de estudio. Se hizo una primera discriminación por títulos y resúmenes, y luego otra de revisión de contenido para descartar aquellos que no cumplía con los criterios mencionados. Vale destacar que el criterio de una década años responde al interés por reportar un cuerpo relativamente actualizado de investigación, asumiendo la pérdida de valiosas investigaciones pioneras publicadas entre 2003 y 2007 (ver Navarro et al., 2016 para un recuento de estos artículos).

Identificados los 24 textos del corpus, se construyó una matriz de datos en Excel con algunas categorías a priori (revista, año, autores, filiaciones, objeto/sujeto de estudio, método y metodología de análisis, ciclos formativos estudiados y $n$ de la muestra). Conforme se realizó la lectura se añadieron otros elementos que resultaban comunes a algunos textos (disciplinas, géneros y años del ciclo estudiados, autores referenciados, hallazgos y conceptos presentados en el marco teórico).

Finalizado este proceso, se identificaron los rasgos extrínsecos de autoría y publicación, y estuvo constituida por: (i) características de la revista de publicación, (ii) autores y (iii) filiaciones institucionales, y (iv) idioma. A continuación se sistematizaron los rasgos intrínsecos, como intereses y hallazgos de investigación por medio de las categorías: (i) marcos de referencia, (ii) áreas y géneros de estudio, (ii) ciclos formativos de interés, y (iv) diseños y muestras. El corpus levantado con estos criterios se detalla en la Tabla 1 a continuación: 


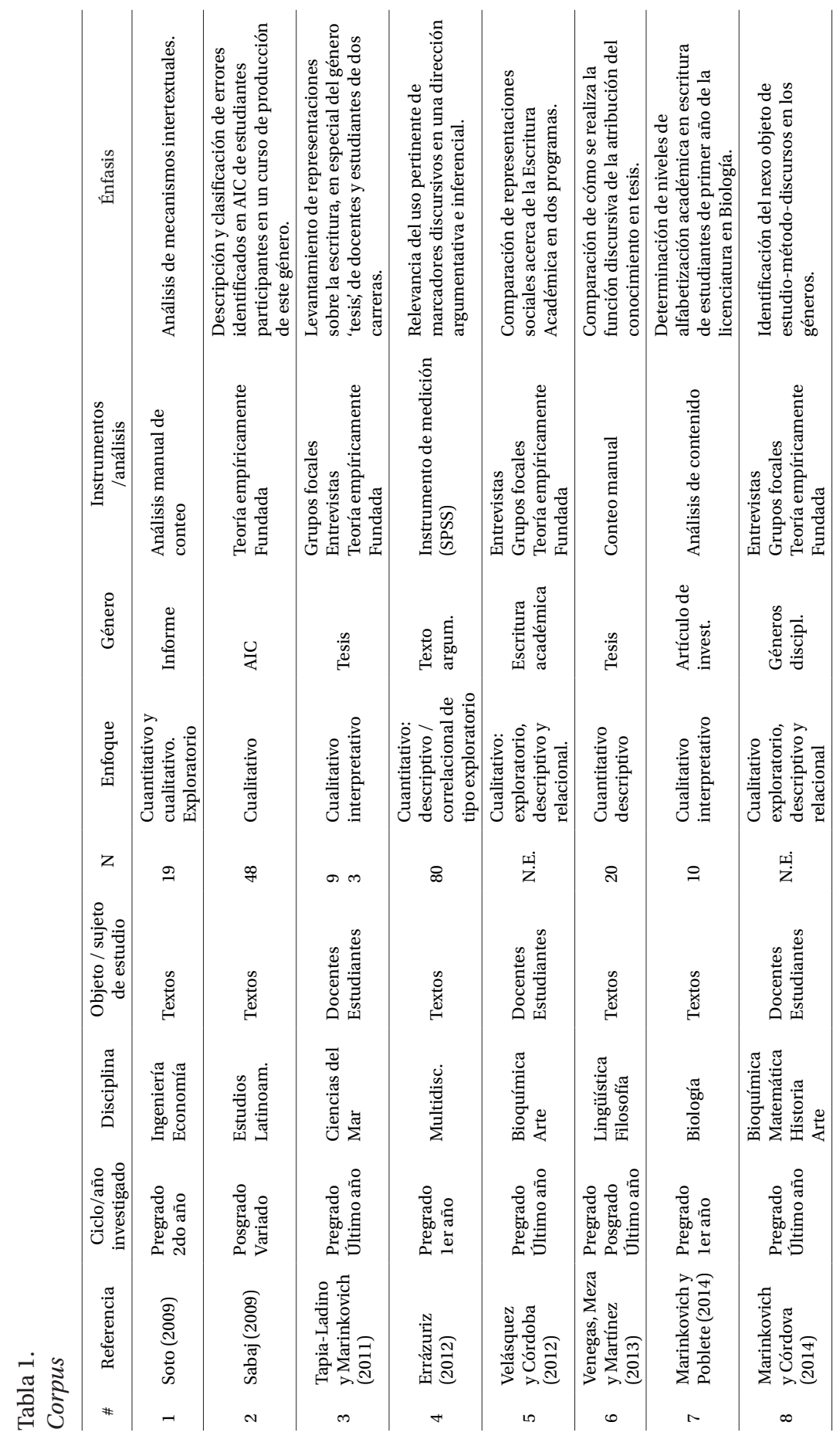




\begin{tabular}{|c|c|c|c|c|c|c|c|}
\hline 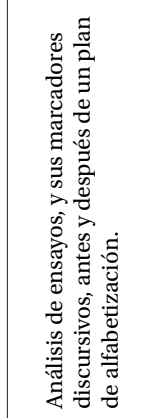 & 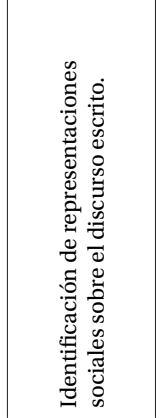 & 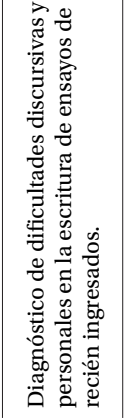 &  & 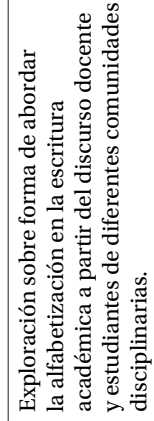 & 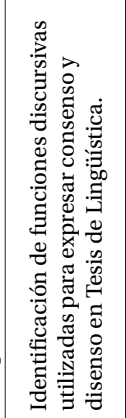 & 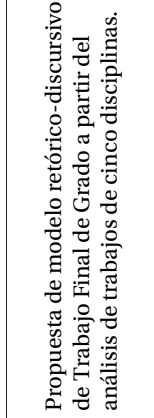 & 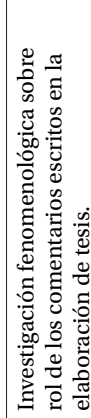 \\
\hline 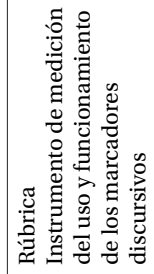 & 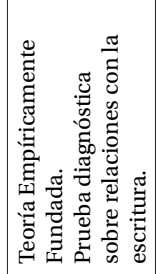 & 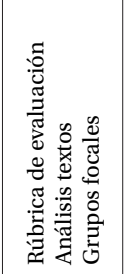 & 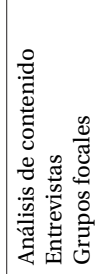 & 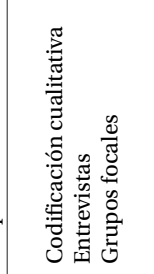 &  & 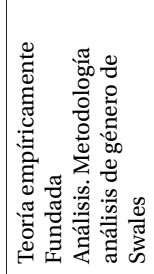 & 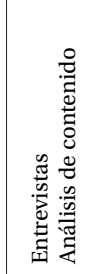 \\
\hline 蓠 &  & 节 & 惑 & 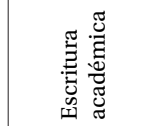 & 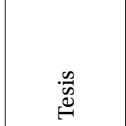 & 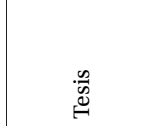 & 悉 \\
\hline  & 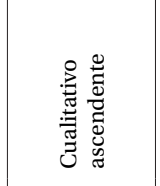 & 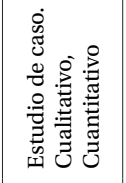 & 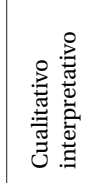 & 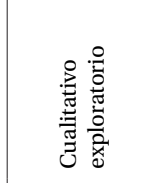 & 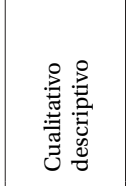 & 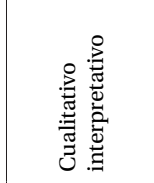 & 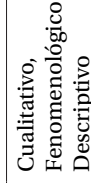 \\
\hline 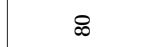 & in & ○ & $\simeq \mathcal{F}$ & $\stackrel{\leftrightarrow}{N} 0$ & $m$ & & \\
\hline 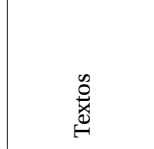 & 总 & 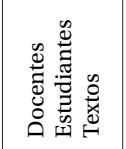 &  & 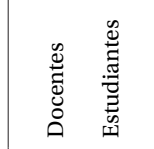 & 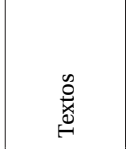 & 递 &  \\
\hline 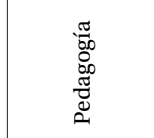 & 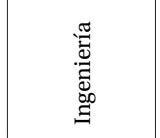 & 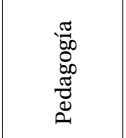 & 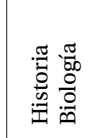 & 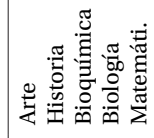 & 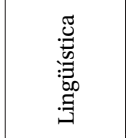 & 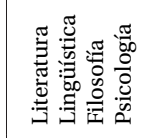 &  \\
\hline 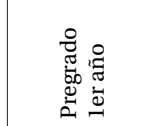 & 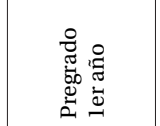 & 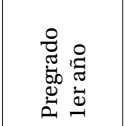 & 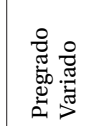 &  & 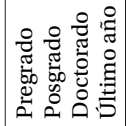 & 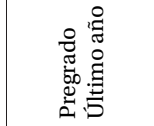 & 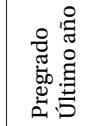 \\
\hline 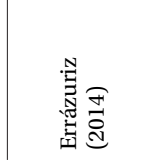 & 密 & 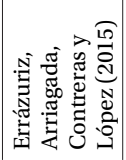 & 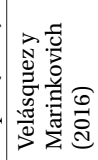 & 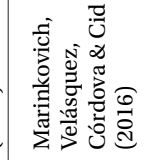 & 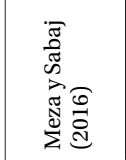 & 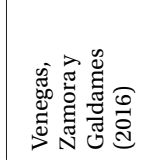 & 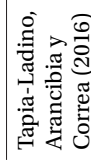 \\
\hline & $=$ & $=$ & & - & & & \\
\hline
\end{tabular}









\section{Resultados y discusión}

\section{Dimensiones extrínsecas}

Sobre la frecuencia de publicación, en 2008 y 2010 no se hallaron investigaciones que cumplieran con los criterios de búsqueda, mientras que los años 2016 y 2017 presentan la mayor cantidad de publicaciones. Adicionalmente, todos estos estudios, excepto dos (Marinkovich, Velásquez, Córdova \& Cid, 2016; Marinkovich, Sologuren \& Shawky, 2018), fueron escritos en español, y el corpus completo está publicado en diez revistas latinoamericanas y una española, con una frecuencia ilustrada en la Figura 1:

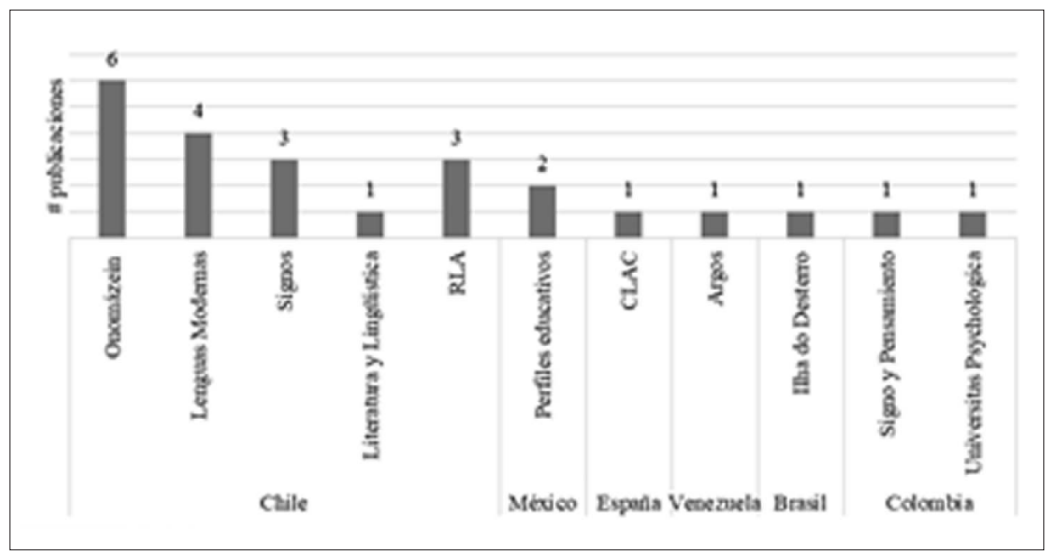

Figura 1. Revistas y frecuencia

Fuente: Elaboración propia

Asimismo, el grueso del corpus fue producido por uno (\#1, 2, 4, 9, 10 y 18), dos (\#3, 5, 7, 8, 12, 14, 17 y 22) o tres autores (\#6, 15, 16, 19, 20, 21 y 23), un par incluye cuatro (\#11 y 13), y solo uno incluye cinco autores (\#24). Ninguno presenta alianzas con investigadores internacionales ni establece análisis comparativo con instituciones de educación superior fuera de Chile. El corpus agrupa 33 investigadores (autores y coautores) afiliados a un total de seis universidades (ver Figura 2): 


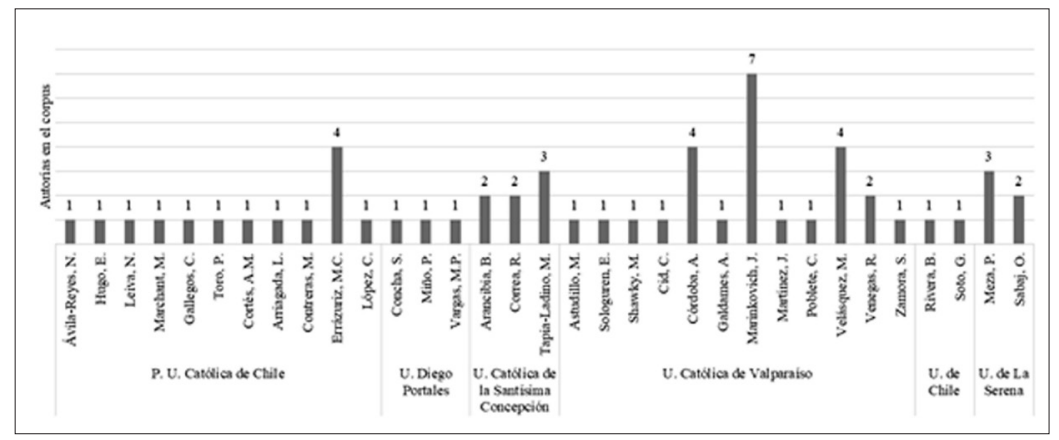

Figura 2. Autores por recurrencia y filiación institucional.

Fuente: Elaboración propia

La preferencia idiomática y la selección de revistas favorece el diálogo regional y local. De ello resulta destacable el compromiso de las revistas chilenas con estudios nacionales, así como el interés de los autores por compartir estas experiencias internamente. No obstante, superar fronteras idiomáticas, regionales y locales, e incluso considerar sinergias con investigadores internacionales, contemplar diseños comparativos y ofrecer discusiones desde contextos distintos, puede contribuir a la participación en el diálogo en y con países cuya experiencia y paradigmas constituyen la base de las tradiciones latinoamericanas.

Por otro lado, el número de filiaciones institucionales referidas es acotado si se pretende poner en perspectiva la alfabetización académica chilena. El perfil de las universidades involucradas da cuenta de una pequeña parte del universo de estudiantes chilenos en formación superior, y sus características difieren de las de otras instituciones nacionales. Este vacío puede responder a una etapa aún temprana del estado de desarrollo de estudios nacionales en el área y a una masa crítica relativamente pequeña.

\section{Dimensiones intrínsecas}

\section{Marcos de referencia}

Para identificar los autores más referenciados en el corpus, se revisó la lista bibliográfica de cada uno de los estudios analizados y se rescataron aquellos que se encontraban incluidos en cuatro o más investigaciones del corpus. Importante señalar, por un lado, que en referencias 
con más de un autor, se tuvo en cuenta únicamente al primero; y, por otro, que en los casos en que se hubieran referenciado varias obras de un autor, este se contó una sola vez. Así mismo, se excluyó la autocitación cuando en una referencia se mencionara al menos a uno de los autores del estudio analizado, aunque no se tratase del principal. La figura 3 a continuación presenta la recurrencia de los autores por obras según su mención en las referencias de los artículos analizados.

La presencia de varios de los autores internacionales identificados en las referencias del corpus coincide con lo reportado por diversos estudios en textos latinoamericanos (Ávila Reyes, 2017; Bazerman et al., 2016; Tapia Ladino et al., 2016), por lo que se constata que estos autores representan una suerte de núcleo conceptual de la disciplina. La alusión frecuente a la autora Paula Carlino es consistente con la recurrente mención, en varios de los marcos teóricos de corpus, al creciente interés regional y local en materia de alfabetización académica, y se vincula con la acuñación del término "alfabetización académica". Frecuentemente, esta se define desde una lógica de procesos de enculturación en géneros discursivos y comunidades discursivas variadas y nuevas para los recién ingresados, quienes deben enfrentarse a nuevos contextos que movilizan una "nueva alfabetización" (Marinkovich y Poblete, 2014); o a un proceso de aculturación mediante el que se accede a nuevas prácticas (Córdova, 2015).

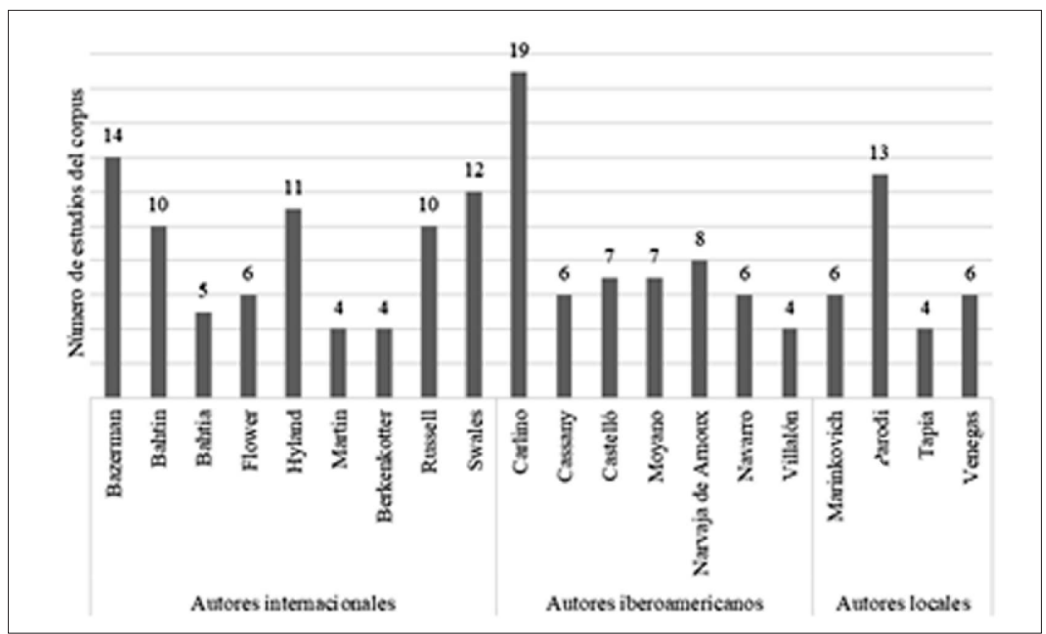

Figura 3. Referencias de mayor citación en bibliografía del corpus.

Fuente: Elaboración propia 
Se constata, así, un cierto acuerdo en la definición de la escritura académica en términos de la entrada a prácticas culturales situadas en mundos sociales. Del mismo modo, también destaca un acuerdo en reconocer el lenguaje no solo como herramienta de utilización transversal en las disciplinas sino también desde su potencial epistémico, y la importancia de su desarrollo en la universidad (Errázuriz, Arriagada, Contreras y López, 2015; Córdova, 2015; Marinkovich y Poblete, 2014; Velásquez y Marinkovich, 2016), y su relación con los géneros discursivos (Marinkovich y Córdova, 2014).

En términos generales, desde la teoría, pareciera haber un consenso entre los autores respecto de la escritura como un instrumento que trasciende a la reproducción y que anida un potencial cognitivo importante; así como la alfabetización académica como proceso de familiarización con nuevas comunidades o géneros discursivos que les son desconocidos a los recién ingresados a la universidad, conceptos que aparecen a través de textos que responden en diferente medida a diferentes paradigmas sobre la naturaleza de la escritura.

\section{Géneros y áreas estudiadas}

Otro elemento que destaca es la inclinación por estudiar las áreas de Humanidades, Ciencias Naturales y Ciencias Sociales, cuya recurrencia se ilustra en la Figura 4:



Figura 4. Áreas disciplinares estudiadas.

Fuente: Elaboración propia 
Aunque desde un foco distinto, los hallazgos coinciden con los de Navarro et al. (2016) en una preferencia por las Humanidades y Ciencias Sociales, y para el caso chileno cabe añadir también las Ciencias Naturales. Esta tendencia puede responder, primero, a los vínculos directos ya instalados de los investigadores, y por tanto su acceso a estudiantes y productos de estas disciplinas; y, segundo, al imaginario de que estas áreas requieren del ejercicio escrito más que otras. En una línea adyacente, Chois y Jaramillo (2016), en su estado del arte sobre investigaciones relacionadas con posgrado, señalaron las Ciencias Sociales y Humanas como áreas más abordadas por los estudios analizados en su corpus. En más detalle, los mismos autores identificaron que 16 de los 39 proyectos tuvieron lugar en programas de educación, lo que es equivalente a lo encontrado en el presente estudio; seis de los 23 productos trabajan con programas de pedagogía (\#9, 11, 16, 17, 18 y 21) como la disciplina más frecuente.

En cuanto a los géneros estudiados, la tesis es el más recurrente (\#6, 14, 15, 21 y 22). El estudio de Tapia-Ladino, Correa y Arancibia (2017) analiza los comentarios del tutor como género dentro de otro género y destaca su rol en el ajuste de la tesis a las convenciones discursivas que le son propias. Otros estudios sugieren variaciones entre disciplinas tanto en términos de organización retórica de la tesis (Venegas, Zamora y Galdames, 2016) como de las representaciones sociales sobre esta (Venegas, Meza y Martínez, 2013; Tapia-Ladino y Marinkovich, 2011). Más aún, dentro de una misma disciplina se identificaron divergencias de percepción (Venegas, Meza y Martínez, 2013) según el ciclo formativo (pregrado/posgrado) (Venegas, Meza y Martínez, 2013), así como de uso de intertextualidad (Meza y Sabaj, 2016) y funciones para comunicación del conocimiento propio y ajeno (Meza y Rivera, 2018). Igualmente, Concha, Miño y Vargas (2017) registraron poco consenso entre las representaciones sobre géneros discursivos entre miembros de una misma comunidad.

Otros estudios reafirman, desde las concepciones sobre la escritura, este disenso entre disciplinas. Velásquez y Córdoba (2012) encontraron diferencias importantes entre las representaciones sociales sobre escritura académica de estudiantes y docentes de Bioquímica frente sus homónimos en Arte; y Marinkovich et al. (2016) localizaron discrepancias en la concepción del potencial epistémico de la escri- 
tura, y de su orientación (formativa/prescriptiva) entre cinco disciplinas. Además, se halló utilización de distintos géneros en el proceso de formación según áreas (Velásquez y Marinkovich, 2016; Marinkovich, et al., 2016), aunque algunos, como el ensayo o el informe, aparecen como transversales (Marinkovich et al., 2016). En el caso del trabajo de Ávila y Cortés (2017), se encontraron coincidencias en lo que tanto estudiantes como docentes consideraban dificultades propias de la escritura de informes de caso.

\section{Ciclos formativos de interés}

Se identificó en el análisis del corpus una predominancia de la investigación de escritura académica en pregrado por sobre posgrado y doctorado, pues únicamente el estudio de Sabaj (2009) se centra en este nivel; Meza y Sabaj (2016), analizan comparativamente las tesis de Lingüística de pregrado, posgrado y doctorado; y Meza y Rivera (2018) analizan comparativamente recursos discursivos en la comunicación del conocimiento propio en tesis de pregrado, magíster y doctorado. En este sentido, seis de los estudios se ocupan del primer año de carrera ( $\# 4,7,9,10,11$ y 24), diez de último año ( $\# 3,5,6,8,14,15,16,21$, 22 y 23), y los siete restantes ocupan años distintos o bien combinan el análisis de diferentes años. Lo anterior contrasta con lo explicitado por Navarro et al. (2016), quienes, pese a señalar una preferencia regional por el pregrado, puntualizaron que las investigaciones latinoamericanas publicadas en la revista chilena Signos balanceaban su interés por diferentes niveles educativos.

La recurrencia del estudio de los ingresantes es comprensible bajo la premisa, que ya se ha relevado en este mismo grupo de artículos, de que los estudiantes se enfrentan a una comunidad discursiva desconocida, de manera que es fundamental identificar y trabajar en los principales retos que presenta este proceso de enculturación. Errázuriz explica su elección señalando que "en tanto los estudiantes no hayan sido preparados para el contexto académico, son los docentes quienes deben iniciarlos en la cultura letrada de su disciplina en todas las cátedras del currículo" (2017, p.42), similar a lo explicitado por Marinkovich y Poblete, quienes afirman que la alfabetización académica no es un "estado" ni es un único conjunto de habilidades que sirvan para siempre (2014). 
Sin embargo, pese a la explicitación de un enfoque cultural de la escritura, varios de los estudios centrados en el primer año de pregrado -cinco de los cuales analizan solo textos- puntualizan diversas dificultades de los ingresantes, como carecer de bases para la realización de textos argumentativos (Errázuriz, Arriagada, Contreras y López, 2015), usar marcadores discursivos de forma limitada (Errázuriz, 2012; 2014), desconocer los procedimientos del ejercicio escrito (Córdova, 2015), estar en un nivel inicial de alfabetización académica en dimensiones científicas y escriturales (Marinkovich y Poblete, 2014) o reproducir de manera imprecisa el contenido de los textos citados (Hugo, Leiva, Marchant, Gallegos y Toro, 2018). Esta constatación de un déficit (Russell, 2002; Zavala, 2011) como parte de la justificación de un estudio es concordante con la prevalencia de los regímenes de evaluación en el estudio de la escritura, descritos por autores como Lillis (2017), quien plantea que algunas categorías analíticas utilizadas en la investigación de la escritura pueden reflejar un normativismo implícito. Esta evaluación puede incluso llevarse a los resultados de estudios que constatan imprecisiones o imperfecciones en las formas escritas de estudiantes y que son llamativamente prevalentes en la literatura sobre escritura estudiantil.

Dentro de las investigaciones con enfoque cognitivo halladas por Ortiz (2011), también resulta frecuente una perspectiva sobre los procesos cognitivos de los estudiantes expresada en términos de déficit. En el presente análisis se identificó una correspondencia entre esta afirmación y algunos de los estudios del corpus. De este modo, algunos autores sintetizan en la introducción o el marco teórico algunas de las dificultades en escritura que poseen los ingresantes a la educación superior, como el paso de la dimensión reproductiva a la función epistémica, el reconocimiento de un lector, y su inexperiencia en términos de producción escrita, lo que genera argumentaciones débiles y textos vagos. Tal preocupación sobre el recién ingresado responde, por ejemplo, a resultados de pruebas estandarizadas de medición de la escritura. Estas ideas presentes en los textos tensionan el supuesto de la alfabetización académica de que el ingreso a la universidad implica la familiarización con prácticas nuevas para los estudiantes pero, sobre todo, impone ciertos valores implícitos de la corrección que pueden resultar en una visión uniformizada y poco sensible a la diversidad de 
experiencias que se plasman en la escritura ( $\mathrm{Lu}, 1991)$ y dejar fuera el estudio de los incomes o aportes de los alumnos a las experiencias de aprendizaje universitario (Eodice, Geller \& Lerner, 2016), temas particularmente sensibles en el contexto de inclusión actual de la universidad latinoamericana (Pérez y Natale, 2017).

Por otro lado, el interés por los estudiantes de último año de pregrado se ve justificado a partir de una preocupación por conocer el estado de la culminación del proceso de formación profesional. Como respuesta a ello, del corpus hallado, casi la totalidad de estudios centrados en último año refleja este interés mediante estudios en torno al trabajo de grado -o tesis-, lo que cobra sentido "debido a su rol fundamental en la difusión y ratificación del conocimiento, así como también en la persuasión a la comunidad académica para aceptar un nuevo integrante" (Meza y Sabaj, 2016, p.389). En otros términos, "el género tesis es una de las instancias textuales-comunicativas más importantes que el estudiante tiene para acreditar el conocimiento propio de su comunidad, haciendo uso de las formas propias de las prácticas discursivas de su disciplina" (Venegas, Meza y Martínez, 2013, p.156); es decir, una propensión por constatar la manera como se ha materializado este proceso de enculturación.

Por último, se detecta un importante vacío de investigación en estudios a nivel de postgrado, pues solo uno de los textos atiende este nivel (Sabaj, 2009), lo que lo que coincide con los hallazgos respecto de Chile en revisiones específicas sobre este tema (Chois y Jaramillo, 2016).

\section{Diseños y muestras}

A lo largo del corpus se tiende a trabajar con muestras medianas o pequeñas y métodos cualitativos (17 estudios), con acotados estudios cuantitativos ( 5 estudios) y dos que utilizan ambos métodos, aunque sin declararse mixtos. En los cualitativos impera el análisis de contenido y la combinación de procesos inductivos y deductivos; mientras que solo dos estudios (Errázuriz, 2014; Sabaj, 2009) contemplan en su diseño una intervención educativa.

Respecto del objeto de estudio, hay división entre análisis textual y estudio de sujetos: 12 y 9 estudios, respectivamente, y tres adicionales (Errázuriz et al., 2015; Ávila y Cortés, 2017; Marinkovich et al., 
2018) que incluyen el examen tanto de textos como de participantes. Esta polarización de los objetos/sujetos de estudio en el corpus coincide con lo concluido por Ortiz (2011) y Ávila (2017). La primera autora hizo hincapié en los dos enfoques registrados a lo largo del corpus que analizó, a saber, uno cognitivo, en cuyos estudios son comunes las propuestas de modelos, técnicas y estrategias para la elaboración de textos; y otro sociocognitivo, dentro el cual el contexto constituye un aspecto fundamental del proceso de producción, de manera que tales estudios se centran en la escritura como práctica social y cultural. En el segundo estudio, se identificó la existencia de una dicotomía entre los estudios latinoamericanos: centrados en los textos, desde el análisis del discurso académico como producto (coincidente con hallazgos de Navarro et al. (2016)); o enfocados en enseñanza y aprendizaje, desde los procesos (Ávila, 2017). Esta tensión es interesante de ser analizada en términos del paradigma o las concepciones sobre la naturaleza de la escritura que implican. En efecto, Lillis \& Scott (2007) explicitan que una investigación demasiado centrada en el producto textual, lo que ellas denominan "sesgo textual", puede informar políticas y planes de formación abrumadoramente textualistas, que pasen por alto las necesidades de aprendizaje y los saberes de los escritores en los nuevos contextos formativos.

En los estudios que relevan información sobre las creencias y percepciones de los involucrados en el proceso, las entrevistas y los grupos focales se identificaron como las técnicas más comunes de recolección de datos. Tal recurrencia sugiere una posición desde la cual se considera que, para generar un cambio, resulta importante atender a las ideas sobre la escritura que tienen los miembros de la comunidad. Esto, a su vez, devela una afinidad con la comprensión del fenómeno desde una perspectiva fenomenológica, que deja de lado las generalizaciones y posturas políticas tradicionales desde las que se ha planteado el término (Street, 2010), para comprenderlo desde las variaciones de contexto y cultura.

Por su parte, Tapia-Ladino y Marinkovich (2011) explican la elección de grupos focales y entrevistas: "para realizar cambios educativos profundos en esta materia, es necesario conocer cómo vive una comunidad una tarea de escritura, qué valor le asignan, bajo qué supuestos enfrentan el proceso de enseñanza, qué causas le atribuyen 
al desempeño, entre otras interrogantes" (p. 279). Tal intención de acción a partir de las construcciones sociales no dista de la de Velásquez y Córdova (2012), quienes señalan que "no debe olvidarse que cada comunidad tiene sus propias concepciones, géneros propios y convenciones que es necesario sacar a la luz, antes de proponer algunas ideas en cuanto a planes de intervención educativa para los estudiantes" (p. 5). Posicionamientos como estos explican naturalmente la selección de diseños de corte inductivo, a partir de los cuales "se busca conocer patrones y conjuntos de características que permitan otorgarle una estructura o modelo al fenómeno, sin caer en la integración teórica previa" (Velásquez y Marinkovich, 2016) de tal manera que se conduzca a modelos emergentes de las comunidades discursivas; o bien la inclinación por el uso de la Teoría Empíricamente Fundada, que "implica un método comparativo de datos, que opera de manera inductiva y busca desarrollar una teoría para explicar el cómo y por qué de un fenómeno en particular" (Marinkovich y Córdova, 2014, p.46). Esta última teoría (TEF) resulta ser igualmente recurrente en análisis textuales entre los hallados en el corpus (Venegas, Zamora y Galdames, 2016; Meza y Sabaj, 2016; Sabaj, 2009). A partir de ello se puede dar cuenta de una concepción dinámica y contextualizada del género, incluso cuando el foco de estudio es lo textual.

De este modo, trabajos como el de Tapia-Ladino, Arancibia y Correa (2016) optan por reconocer la importancia de la retroalimentación en el entendido de que el uso de los comentarios escritos expresa una dinámica dialógica que revela relaciones sociales, procesos de aculturación y de enseñanza aprendizaje. Córdova (2015), por su parte, pesquisa representaciones sociales a partir del discurso escrito de los estudiantes de ingeniería, relevando solo aquellas que se reiteran en el grupo.

En virtud de lo mencionado queda explicada también la utilización de muestras relativamente pequeñas, desde menos de 10 participantes hasta alrededor de 50, para hablar de inferencias o descripciones de comunidades discursivas dentro de contextos particulares. En términos de Ortiz (2011), este enfoque se relaciona con un reconocimiento de tensiones y transformaciones que posibilita la creación de ambientes con condiciones para lograr cambios significativos en las prácticas educativas. Resulta interesante la recurrencia de tal preocupación en 
Chile frente a los pocos estudios a nivel internacional sobre escritura que se enfocan en las representaciones sociales y percepciones de los sujetos involucrados en los procesos de enseñanza y aprendizaje.

Por otro lado, dentro de las investigaciones que se ocupan del análisis textual, se registran otros intereses, como la intertextualidad (Soto, 2009; Venegas, Meza y Martínez, 2013; Meza y Sabaj, 2016; Hugo et al., 2018); elementos discursivos tales como el uso de marcadores (Errázuriz, 2012; 2014); aspectos ortográficos y argumentativos (Errázuriz, Arriagada, Contreras y López, 2015); y errores frecuentes de escritura (Sabaj, 2009). Para Errázuriz, la importancia del análisis del uso de marcadores discursivos redunda en que "estos cumplen un rol clave en la orientación de las inferencias y en el procesamiento de la información en la lectura y, especialmente, en el proceso de producción de textos" (2014, p.219), mientras que tanto Soto (2009) como Hugo y equipo (2018) coinciden en que un manejo adecuado de la intertextualidad es un elemento clave dentro del discurso especializado y da cuenta de la comprensión gradual del conocimiento disciplinar.

\section{Consideraciones finales}

La difusión de artículos nacionales sobre "alfabetización académica" entre 2008 y abril de 2019 en revistas de alto impacto está representada por 24 estudios que indican una consolidación de este interés, con mayor afluencia en los años 2016 y 2017. La totalidad de los estudios encontrados se encuentra publicada en revistas latinoamericanas, primordialmente en castellano, y firmada por un número acotado de autores e instituciones. Esta preferencia por la consolidación de un diálogo local resulta atingente desde el punto de vista de la resistencia a las dinámicas de publicación anglocéntricas (Curry y Lillis, 2017), y puede comprenderse como una fortaleza del ámbito. Sin embargo, la concentración de la producción científica empírica e indexada en solo un conjunto acotado de instituciones debería encender las alarmas con respecto a la centralización del sistema académico chileno. Por último, pese al valor y la importancia de la producción académica local, es necesario también desarrollar estrategias para unirse a las conversaciones internacionales en calidad de productores de conocimientos y de teorías, en lo que Donahue (2009) ha denominado un intercambio intelectual igualitario entre culturas o equal exchange. 
Con respecto a los marcos teóricos y antecedentes, se evidencia un alineamiento con los principios generales de "alfabetización académica" según Carlino (2003), la autora más citada en el corpus. Adicionalmente, se identificaron algunos puntos comunes que se consideran importantes a la hora de aventurar una caracterización de la producción chilena sobre la temática, como la alusión a los procesos de enculturación en géneros discursivos y comunidades discursivas que son nuevas para los estudiantes, y el reconocimiento del potencial epistémico de la escritura.

En primer lugar, hay un interés predominante en el estudio de carreras inscritas en Humanidades, Ciencias Naturales y Ciencias Sociales, muchas veces de manera comparativa entre disciplinas. Los hallazgos señalan la identificación de variaciones disciplinares en cómo se entienden, construyen y estructuran los géneros. Igualmente, el análisis del corpus arrojó que se suelen abordar investigaciones sobre el ciclo de pregrado, en detrimento de una atención por los programas posgraduales, lo que indica un vacío de conocimientos que es importante de relevar, considerando el creciente interés sobre este tema (Simpson, Caplan, Cox \& Phillips, 2016).

Un segundo elemento que resultó evidente redundó en el interés preponderante en el estudio de productos o procesos de estudiantes de primer o último año del ciclo, en el estudio del tipo textual argumentativo o pruebas diagnósticas para los nuevos estudiantes; y el género tesis para los prontos a finalizar. A propósito del interés por indagar sobre los recién ingresados, en algunos estudios se localizaron ciertas ideas que dialogan con un paradigma del déficit, propio de los llamados "regímenes de evaluación normativa" de la escritura (Lillis, 2017), que se contradice con los marcos declarados propios de la alfabetización académica y los procesos de enculturación. Adicionalmente, es importante tener en cuenta el potencial efecto de un sesgo textualista, en particular, al propender a dejar fuera de la investigación la experiencia de los escritores ( 12 de 24 trabajos), lo que podría informar políticas y prácticas que también lo hagan.

Como tercer punto, se halló una tendencia a muestras relativamente pequeñas, diseños cualitativos y el uso de entrevistas y grupos focales como técnicas comunes de recolección de datos. Ello es coherente con objetivos de investigación concernientes a la relevación de repre- 
sentaciones sociales, teorías implícitas y percepciones de los actores involucrados. En la misma línea, se corroboró lo señalado en estudios previos en relación a intereses de investigación segmentada en dos tendencias, una concerniente con el análisis textual y cognitivo; y otra vinculada con un interés por las prácticas, estudio de sujetos, y de corte sociocognitivo.

Con respecto a las dinámicas de participación internacional y predominancia del idioma español en los artículos, se considera que la tendencia anglocéntrica de las revistas científicas (Curry \& Lillis, 2017) constituye una barrera para figurar en una mayor variedad de revistas relevantes para la disciplina a nivel internacional. Mientras esto hiere el interés por participar de la producción mundial de conocimientos de manera igualitaria (Donahue, 2009; Martins, 2015), también representa una limitación del presente estudio. Trabajar con índices mainstream redunda en la omisión de resultados en revistas hispanohablantes de menor impacto internacional, pero que aportarían la construcción de un panorama sólido sobre las prácticas investigativas de alfabetización académica chilena para ofrecer una mirada más integral.

Finalmente, se espera que el escenario presentado fortalezca el diálogo y las prácticas nacionales, y nutra las posibilidades de actuación respecto de los procesos de alfabetización académica de los profesionales en formación. Por un lado, es importante seguir cautelando una coherencia teórica entre un modelo de enculturación con uno implícitamente normativo, paradigma del que resulta necesario volvernos conscientes como investigadores (Lillis, 2013). Por otro, conviene que futuras investigaciones empíricas atiendan también ciclos de pregrado, amplíen la caracterización sociodemográfica e institucional contemplada hasta ahora y aborden diseños que trasciendan los diagnósticos de un momento temporal para ahondar en seguimientos sistemáticos del desarrollo escrito en la formación superior que contribuyan a la generación de conocimiento agregable.

\section{Referencias bibliográficas}

Ávila, N. (2017). Postsecondary writing studies in Hispanic Latin America: Intertextual dynamics and intellectual influence. London Review of Education, 15(1), 21-37. https://doi.org/10.18546/ LRE.15.1.03 
Ávila, N. (2018). Locales, regionales y cosmopolitas: análisis intertextual de artículos sobre escritura universitaria en América Latina Hispánica. In R. Celi (Ed.), Escrita na Universidade: panoramas e desafios na América Latina (pp. 47-83). João Pessoa: Editora UFPB-Ideia.

Ávila, N. \& Cortés, A. (2017). El género "informe de caso" en la formación inicial docente: una aproximación basada en la actividad. Lenguas Modernas, 50(2), 153-174.

Ávila, N., González, P. \& Peñaloza, C. (2013). Creación de un programa de escritura en una universidad chilena: estrategias para promover un cambio institucional. Revista Mexicana de Investigación Educativa, 18(57), 357-560. Recuperado de: http://www. scielo.org.mx/pdf/rmie/v18n57/v18n57a10.pdf

Bazerman, C., Dean, C., Early, J., Lunsford, K. J., Null, S., \& Stansell, A. (2012). International Advances in Writing Research: Cultures, Places, Measures. Anderson, South Carolina: The WAC Clearinghouse, Parlor Press.

Bazerman, C., Ávila, N., Bork, A., Poliseli-Corrêa F., Cristovão, V., Tapia-Ladino, M. \& Narváez-Cardona, E. (2016). Intellectual orientations of studies of higher education writing in Latin America. En S. Plane, C. Bazerman, F. Rondelli, C. Donahue, A. Applebee, C. Boré, C., P. Carlino, L. Marquillo, P. Rogers \& D. Russell (Coord.), Recherches en écritures: regards pluriels (pp.281-297). Lorraine, Francia: Université de Lorraine.

Carlino, P. (2003). Alfabetización académica: un cambio necesario, algunas alternativas posibles. Tercer encuentro «La universidad como objeto de investigación», 6, 409-420.

Castelló, M. (2002). De la investigación sobre el proceso de composición a la enseñanza de la escritura. Revista Signos, 35(51-52), 149162. https://doi.org/10.4067/S0718-09342002005100011

Chois, P. \& Jaramillo, L. (2016). La investigación sobre la escritura en posgrado: estado del arte. Lenguaje, 44(2), 227-259.

Concha, S., Miño, P. \& Vargas, M. (2017). Representaciones sociales sobre el conocimiento y la escritura en el pregrado en dos comunidades discursivas: implicancias para la enseñanza de la escritura en Educación Superior. Lenguas Modernas, 50, 109-130.

Córdova, A. (2015). ¿Qué es escribir para estudiantes ingresantes a la carrera de Ingeniería Civil? Un acercamiento a través de las representaciones sociales. Onomázein, 31, 20-37. 
Curry, M. J., \& Lillis, T. M. (2017). Global academic publishing: Policies, perspectives and pedagogies. Bristol: Multilingual Matters.

Donahue, C. (2009). "Internationalization" and Composition Studies: Reorienting the Discourse. College Composition and Communication, 61(2), 212-243.

Eodice, M., Geller, A., \& Lerner, N. (2016). The meaningful writing project. Learning, teaching and writing in higher education. Boulder, Colorado: University Press of Colorado.

Errázuriz, M.C. (2012). Análisis del uso de los marcadores discursivos en argumentaciones escritas por estudiantes universitarios. Perfiles Educativos, 34(136), 98-117.

Errázuriz, M.C. (2014). El desarrollo de la escritura argumentativa académica: los marcadores discursivos. Onomázein, 30, 217-236.

Errázuriz, M.C. (2017). Teorías implícitas sobre la escritura académica en estudiantes de programas de formación inicial docente: ¿inciden en el desempeño escrito? Signo y pensamiento, 36(71), 36-52.

Errázuriz, M.C., Arriagada, L., Contreras, M. \& López, C. (2015). Diagnóstico de la escritura de un ensayo de alumnos novatos de Pedagogía en el campus Villarrica UC, Chile. Perfiles Educativos, 37(150), 76-90.

Comisión Nacional de Ciencia y Tecnología, s/f. Grupos de estudios. Disponible en: http://www.conicyt.cl/fondecyt/grupos-deestudios/

González, C. (2015). Perspectivas y desafíos de la docencia en la Educación Superior Chilena. En A. Bernasconi (Ed.), La educación superior de Chile. Transformación, desarrollo y crisis. (pp. 371-407). Santiago de Chile: Ediciones UC.

Haswell, R. H. (2005). NCTE/CCCC's Recent War on Scholarship. Written Communication, 22(2), 198-223. https://doi. org/10.1177/0741088305275367

Hugo, E., Leiva, N., Marchant, M., Gallegos, C. -\& Toro, P. (2018). Intertextualidad manifiesta en textos de estudiantes universitarios. Caracterización de las citas en una etapa de formación académica inicial. Onomázein, 41, 29-56.

Lillis, T. \& Scott, M. (2007). Defining academic literacies research: issues of epistemology, ideology and strategy. Journal of 
Applied Linguistics, 4(1), 5-32. https://doi.org/10.1558/japl. v4i1.5

Lillis, T. M. (2013). The sociolinguistics of writing. Edinburgh: Edinburgh University Press.

Lillis, T. M. (2017). Resistir regímenes de evaluación en el estudio del escribir: hacia un imaginario enriquecido. Signo y Pensamiento, 71, 66-81. https://doi.org/10.11144/Javeriana. syp36-71.rree

Lu, M.Z. (1991). Redefining the Legacy of Mina Shaughnessy: A Critique of the Politics of Linguistic Innocence. Journal of Basic Writing, 10(1), 26-40. Recuperdo de : https://wac.colostate. edu/jbw/v10nl/lu.pdf

Marinkovich, J. \& Córdova, A. (2014). La escritura en la universidad: Objeto de estudio, método y discursos. Revista Signos, 4(84), 40-63. https://doi.org/10.4067/S0718-09342014000100003

Marinkovich, J. \& Poblete, C. (2014). Alfabetización en escritura académica en Biología: propósitos comunicativos y niveles de alfabetización. Onomázein, 30, 269-285. https://doi.org/ 10.7764/onomazein.30.20

Marinkovich, J., Sologuren, E. \& M. Shawky. (2018). The process of academic literacy in Civil Engineering Computer Science. An approach to academic writing and its genres in a learning community, Círculo de linguística aplicada a la comunicación, 74, 195-220. http://dx.doi.org/10.5209/CLAC.60520

Marinkovich, J., Velásquez, M., Córdova, A. \& Cid, C. (2016). Academic Literacy and Genres in University Learning Communities. Ilha do Desterro, 69(3), 95-113. https://doi.org/10.5007/21758026.2016v69n3p95

Marinkovich, J., Velásquez, M. \& Astudillo, M. (2017). Prácticas de escritura en la comunidad académica de Biología. Caracterización y acercamiento didáctico. Lenguas Modernas, 50, 131-152. Recuperado de: https://revistas.uchile.cl/index. $\mathrm{php} / \mathrm{LM} /$ article/view/49255

Martins, D. (2015). Transnational Writing Program Administration. Boulder, Colorado: Utah State University Press.

Meza, P \& Rivera, B. (2018). La comunicación del conocimiento propio en tesis: variación entre grados académicos en la sección desarrollo teórico, $R L A, 56(1), 115-138$. http://dx.doi. org/10.4067/S0718-48832018000100115. 
Meza, P. \& Sabaj, O. (2016). Funciones Discursivas de Consenso y Disenso en Tesis de Lingüística. Onomázein, 33, 385-411. http://dx.doi.org/10.7764/onomazein.33.23

Molina, V. (2014). Centros de escritura: una mirada retrospectiva para entender el presente y futuro de estos programas en el contexto latinoamericano. Legenda, 18(8), 9-33.

Natale, L. (2013). Integración de enfoques en un programa institucional para el desarrollo de la escritura académica y profesional. Revista Mexicana de Investigación Educativa, 18(58), 685-707.

Navarro, F. (2017). Estudios latinoamericanos de la escritura en educación superior y contextos profesionales: hacia la configuración de un campo disciplinar propio. Lenguas Modernas, 50, 8-14. Recuperado de https://revistas.uchile.cl/index.php/ $\mathrm{LM} /$ article/view/49247/53097

Navarro, F. (2014). Manual de escritura para carreras de humanidades. Buenos Aires, Argentina: Editorial de la Facultad de Filosofía y Letras Universidad de Buenos Aires.

Navarro, F., Ávila, N., Tapia Ladino, M., Cristivao, V., Moritz, M. E., Narváez, E. \& Bazerman, C. (2016). Panorama histórico y contrastivo de los estudios sobre lectura y escritura en educación superior publicados en América Latina. Revista Signos, 49(1), 100-126. http://dx.doi.org/10.4067/S071809342016000400006

Nystrand, M., Greene, S., \& Wiemelt, J. (1993). Where did Composition Studies Come From? An Intellectual History. Written Communication, 10(3), 267-333. https://doi.org/0803973233

Ortiz, E. (2011). La escritura académica universitaria: estado del arte. Ikala, 16(28), 17-41.

Prior, P., \& Thorne, S. L. (2014). Research paradigms : Beyond product , process , and social activity. In E.M. Jakobs \& Daniel Perrin (Eds.), Handbook of Writing and Text Production (pp. 31-54). Boston, MA: Walter De Gruyter.

Pereira, C. (2006). La lectura y la escritura en el CBC: memoria de la experiencia en la cátedra de Semiología. Paper presented at Primer Congreso Nacional: "Leer, Escribir Y Hablar Hoy." Tandil, Argentina.

Pérez, I. \& Natale, L. (2017). Inclusión en educación superior y alfabetización académica. En L. Natale y D. Stagnaro (Comps.), Alfa- 
betización académica. Un camino hacia la inclusión en el nivel superior (pp.13-46). Buenos Aires, Argentina: Eds UNGS.

Russell, D. (2002). Writing in the academic disciplines: A curricular history (Second Ed.). Carbondale: Southern Illinois University Press.

Sabaj, O. (2009). Descubriendo algunos problemas en la redacción de Artículos de Investigación Científica (AIC) de alumnos de postgrado. Revista Signos, 42(69), 107-127. http://dx.doi. org/10.4067/S0718-09342009000100006

Simpson, S., Caplan, N., Cox, M., \& Phillips, T. (2016). Supporting graduate student writers. Research, curriculum \& program design. Ann Arbor, MI: University of Michigan Press.

Soto, G. (2009). Intertextualidad explícita en textos académicos de estudiantes universitarios. Un estudio exploratorio. Literatura y Lingüística, 20, 141-157. http://dx.doi.org/10.4067/S071658112009000100008

Street, B. (2010). Adopting an ethnographic perspective in research and pedagogy. En C. Coffin, T. Lillis, \& K. O'Halloran (Eds.), Applied Linguistics Methods: A Reader (pps. 201-215). London \& New York: Routledge.

Tapia-Ladino, M., Arancibia, B. M. \& Correa, R. (2016). Rol de los Comentarios Escritos en la construcción de la tesis desde la perspectiva de estudiantes tesistas y profesores guía. Universitas Psychologica, 15(4). http://dx.doi.org/10.11144/Javeriana.upsy15-4.rcec.

Tapia-Ladino, M., Ávila, N., Navarro, F. \& Bazerman, C. (2016). Milestones, disciplines and the future of initiatives of reading and writing in higher education: an analysis from key scholars in the field in Latin America. Ilha do Desterro, 69(3), 189-208. http://dx.doi.org/10.5007/2175-8026.2016v69n3p189

Tapia-Ladino, M. \& Marinkovich, J. (2011). Representaciones sociales sobre la escritura de la tesis en la formación académica inicial en el área de las Ciencias del Mar. Onomázein, 2(24), 273297. https://doi.org/10.1590/S1984-63982013000100008

Tapia-Ladino, M., Correa, R. \& Arancibia, B. (2017). Retroalimentación con comentarios escritos de ajuste al género (CEAG) en el proceso de elaboración de tesis de Programa de Formación de Profesores. Lenguas Modernas, 50, 175-192. http://dx.doi. org/10.4067/S0718-09342019000200242 
Velásquez, M. \& Córdoba, A. (2012). Cultura escrita en las licenciaturas en bioquímica y en arte: un acercamiento a través de las representaciones sociales de profesores y estudiantes. Argos, 29(57).

Velásquez, M. \& Marinkovich, J. (2016). Hacia un modelo explicativo del proceso de alfabetización en escritura académica en las licenciaturas en Historia y Biología. RLA, 54(2), 113-136. http://dx.doi.org/10.4067/S0718-48832016000200006.

Venegas, R., Meza, P. \& Martínez, J. (2013). Procedimientos discursivos en la atribución del conocimiento en tesis de lingüística y filosofía en dos niveles académicos. RLA, 51(1), 153-179. http://dx.doi.org/10.4067/S0718-48832013000100008.

Venegas, R., Zamora, S. \& Galdames, A. (2016). Hacia un modelo retórico-discursivo del macrogénero Trabajo Final de Grado en Licenciatura. Revista Signos, 49(1), 247-279. http://dx.doi. org/10.4067/S0718-09342016000400012.

Zavala, V. (2011). La escritura académica y la agencia de los sujetos. Cuadernos Comillas, 1, 52-66. 